\title{
An Integral Method for Mixing, Chemical Reactions, and Extinction in Unsteady Strained Diffusion Layers
}

\author{
GRÉTAR TRYGGVASON and WERNER J. A. DAHM
}

\author{
Department of Mechanical Engineering and Applied Mechanics (G. T.) and Department of Aerospace \\ Engineering (W. J. A. D.), The University of Michigan, Ann Arbor, MI 48109
}

\begin{abstract}
An integral method is presented for determining the evolution of molecular mixing, finite rate chemical reactions, and local extinction in diffusion layers under the effect of an unsteady strain rate. The partial differential equations governing the reactant, product, and temperature profiles are used to derive ordinary differential equations governing the evolution of moments for the product and temperature profiles and for the reactant gradient profiles. The actual profiles enter these equations only through integral moments resulting from the reaction rate terms (referred to as "reaction integrals"). As a consequence, it is possible to accurately track the evolution of the profile moments, and thereby determine global properties of the layer such as burning rates and extinction conditions, using remarkably simple representations for the actual profiles to evaluate the reaction integrals. Here these profile shapes are specified as self-similar families of curves parameterized by just a few degrees of freedom, which then evolve from the moment equations. Results for combustion in isolated strained diffusion layers, as well as for consumption of a burning fuel strip, are generally within a few percent of the results from finite difference solutions of the full equations.
\end{abstract}

\section{INTRODUCTION}

In many conceptual pictures of turbulent reacting flows, much of the molecular mixing and chemical reaction is viewed as occurring in strained laminar diffusion layers. Indeed, local kinematic considerations suggest that any material surface, such as the interface initially separating two reactants, will deform under the influence of a fluid strain field in such a manner as to continually reorient itself toward lying normal to the most compressive principal strain rate axis. Numerical simulations of Ashurst et al. [1] and Gibson et al. [2] confirm a strong correlation for such alignment, even in strain rate fields that vary widely in space and time, as would be encountered in a turbulent flow. This suggests a canonical picture for an isolated strained diffusion layer in which the interface that initially separated the reactants is locally oriented normal to the most compres- sive principal strain rate axis. Molecular diffusion of the reactants across this interface then occurs in the presence of a time-varying strain rate field $\sigma(t)$. The competing effects of strain and diffusion establish an equilibrium strain-limited molecular diffusion layer thickness $\lambda_{D} \sim(D / \sigma)^{1 / 2}$ closely related to the Batchelor scale. A similar competition between the effects of strain and the diffusion of vorticity establishes a strain-limited vorticity layer thickness $\lambda_{\nu} \sim(\nu / \sigma)^{1 / 2}$, related to the Kolmogorov scale, on which the finest strain rate gradients occur. The ratio of the two diffusivities then establishes the relation between these thicknesses as $\lambda_{D} \sim \lambda_{\nu} \cdot \mathrm{Sc}^{-1 / 2}$, where Sc $\equiv(\nu / D)$ and where owing to the similarity of the two diffusion processes, the proportionality constant should be approximately unity. When the local Damköhler number, $\mathrm{Da} \equiv(k / \sigma)$, with $k$ the specific reaction rate coefficient, is sufficiently large, then imbedded within this strained 
molecular diffusion layer is a reaction layer in which the chemical reactions occur, having a characteristic local thickness $\lambda_{R} \sim \lambda_{D} \cdot \mathrm{Da}^{-1 / 3}$.

Such a physical picture of molecular mixing and chemical reactions forms the foundation for the "coherent flame model" of Marble and Broadwell [3], and is also closely related to various implements of "flamelet" models [4-9]. This view also appears in the strained diffusion layer component of the turbulent shear layer mixing and reaction models of Broadwell and Breidenthal [10], Dimotakis [11], and Broadwell and Mungal [12], and in the jet mixing and reaction model of Broadwell [13]. Of course, as the separation between neighboring layers is continually reduced by the strain field, any such isolated diffusion layer will eventually interact with an adjacent layer. It is then no longer appropriate to view the local mixing and reactions as occurring in distinct layers. Here, we present a simple integral method for determining the evolution of mixing and reactions in such isolated as well as interacting strained molecular diffusion and chemical reaction layers in a turbulent reacting flow.

\section{Background}

The steady-state behavior of mixing and chemical reactions in isolated diffusion layers under the effects of a constant strain rate has been investigated extensively within the simplifying approximation of a one-step overall reaction mechanism. The simplest case, with infinitely fast kinetics, is given by the classic flame sheet solution of Burke and Schumann [14]. For finite rate reactions, Fendel [15] gives the internal structure within the layer in the two asymptotic limits of slow and fast reaction rates. These yield the characteristic $S$ curve of, say, maximum temperature versus Damköhler number for realistically large activation energies (see also Ref. 16). Liñán [17] presents solutions based on large activation-energy asymptotics for the entire range of Damköhler number and focusing in particular on the internal structure in the layer and on the ignition and extinction limits. Using an extensive set of elementary reactions for a more realistic description of the chemical kinetics, Dixon-Lewis et al. [18] give numerical results for the internal structure of such a diffusion layer under a constant strain rate, including results at low Damköhler numbers to determine the extinction conditions.

Considerably less is known about the progress of reactions in such diffusion layers when the strain rate is no longer constant. Carrier et al. [19] (see also Ref. 20) give the general conserved scalar solution for the time-varying strained diffusion layer problem, as well as analytical solutions for the layer structure resulting from a one-step reaction in the limit of infinitely fast kinetics, and asymptotic solutions for the internal structure under fast but finite rate reactions for a few simple time-dependent configurations. The flamelet formulation in Refs. 7 and 8 accounts for effects of an unsteady strain rate history through the scalar dissipation. However, its implementation generally involves the use of a library of quasi-steady flamelets, and this in turn requires that the characteristic time scale on which the strain rate varies must be long compared to the time scale on which the diffusion and reaction layer structure adjusts to the changing strain rate. As Peters [7, 8] points out, this limitation is especially important when the layer is near extinction or reignition. Haworth et al. [21] replace the quasi-steady strain rate in the flamelet library approach with an approximate "equivalent strain rate" that accounts for history effects. Of course, numerical solutions of the detailed internal structure for mixing and reactions in strain diffusion layers with a time-varying strain rate are possible at any chosen level of completeness in representing the chemical kinetics. However, such numerical solutions are far too time consuming, even for relatively simple kinetic representations, to make this approach practical for use in simulations of turbulent reacting flows.

\section{Present Work}

Here we take a fundamentally different approach. Rather than attempting to determine the detailed internal structure within the layer under the effect of an arbitrarily time-varying strain rate, we instead aim to determine only the global layer properties of principal interest, such as local 
burning rates and extinction conditions. To this end, we use the partial differential equations governing the reactant, product, and temperature profiles to derive the ordinary differential equations for the evolution of moments of these profiles. From these, it will be seen that the detailed internal structure affects the layer evolution only through integrals resulting from the reaction rate terms. In view of the integral nature of this dependence, no effort is made to accurately represent this internal structure. Instead, we model the internal structure by assuming the profiles to be self-similar and represented by a family of curves parameterized by a few degrees of freedom. The moment equations then determine the time evolution of these degrees of freedom, which in turn determine the global properties of the layer.

Note that this approach is similar in spirit to integral methods commonly used in determining the development of boundary layers under the effects of arbitrary pressure gradients (e.g., Ref. 22). In that case, the von Kármán momentum integral equation is closely related to an evolution equation for the zeroth moment of the velocity profile. Various integral methods assume that the local velocity profile can be adequately characterized by certain families of shapes with just a few degrees of freedom. The von Kármán equation then determines the downstream evolution of the resulting global properties of the boundary layer such as the wall shear stress, the layer thickness, and the conditions for separation in much the same manner as is done here for strained molecular diffusion and chemical reaction layers.

The presentation is organized as follows. In the following section we formulate the governing equations for the species and temperature profiles in an isolated strained diffusion layer. From these, we derive the evolution equations for the profile moments in the subsequent section, after which we discuss the closure of these equations by imposing simple shapes for the profiles where they appear explicitly. We then present results for various isolated and interacting layers and make comparisons with full finite difference solutions of the governing equations to assess the accuracy of this method. Conclusions regarding the method are given in the final section.

\section{STRAINED DIFFUSION LAYER FORMULATION}

A sufficiently realistic approximation for the purposes of this study is to treat the chemistry as an overall, single-step, binary, irreversible reaction between reactants $A$ and $B$ to yield a product $P$ as

$\nu_{A} A+\nu_{B} B \stackrel{k}{\rightarrow} \nu_{P} P$,

where $\nu_{i}$ are the molar stoichiometric coefficients. Note that this formulation is completely general as to the reactants $A$ and $B$ that are being tracked; they do not need to be pure fuel and oxidizer, and can equally well represent partially premixed reactants. The overall reaction rate is taken to be first order with respect to each of the reactants, and the specific reaction rate coefficient $k$ is taken to be controlled by temperature-dependent Arrhenius kinetics of the form

$k=Z e^{-E / R T}$,

where $Z$ is the pre-exponential frequency factor and $E, R$, and $T$ are the overall activation energy, the universal gas constant and the absolute temperature, respectively. The transport equations [23] for the molar concentrations $c_{i}$ of the species $i=\{A, B, P\}$ and the temperature $T$ can then be written in the form

$$
\begin{aligned}
\left.L\left(c_{i} ; \operatorname{ReSc}\right)_{i}\right)= & \pm \nu_{i} \operatorname{Da} c_{A} c_{B} e^{-Z e / T} \\
& -G\left(c_{i} ; \operatorname{ReSc_{i}}\right), \\
L(T ; \operatorname{Re} \operatorname{Pr})= & \nu_{P} \operatorname{He~Da~} c_{A} c_{B} e^{-Z e / T} \\
& -G(T ; \operatorname{Re} \operatorname{Pr}),
\end{aligned}
$$

where the minus sign is appropriate in Eq. 1 when $i=\{A, B\}$ and the plus sign when $i=$ $\{P\}$. Here

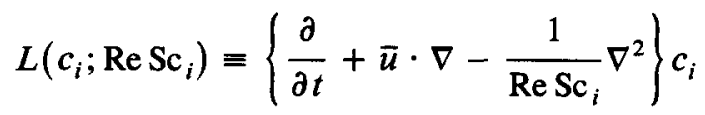

and

$$
\begin{aligned}
& G\left(c_{i} ; \operatorname{ReSc_{i}}\right) \\
& \equiv\left\{\nabla \cdot \bar{u}-\frac{1}{\operatorname{ReSc}}\left(\frac{\nabla D_{i}}{D_{i}} \cdot \frac{\nabla c_{i}}{c_{i}}\right)+\right.
\end{aligned}
$$




$$
\left.\frac{1}{\operatorname{ReSc} c_{i}}\left(\frac{\nabla D_{i}}{D_{i}}+\frac{\nabla c_{i}}{c_{i}}+\nabla\right) \cdot \frac{\nabla \rho}{\rho}\right\} c_{i}
$$

All quantities have been made dimensionless by the reference values $L^{*}, U^{*}, T^{*}, \rho^{*}$, and $c^{*}$ for the length, velocity, temperature, density and molar concentrations, respectively. The resulting dimensionless governing parameters appearing in Eqs. 1 and 2 are given by

$$
\begin{aligned}
& \operatorname{ReSc}_{i} \equiv \frac{U^{*} L^{*}}{D_{i}}, \quad \operatorname{Re} \operatorname{Pr} \equiv \frac{U^{*} L^{*}}{D_{T}}, \\
& \mathrm{Da} \equiv \frac{Z c^{*}}{U^{*} / L^{*}}, \quad \mathrm{Ze} \equiv \frac{E}{R T^{*}}, \\
& \mathrm{He} \equiv \frac{q}{c_{v} T^{*}},
\end{aligned}
$$

where $q$ is the heat release per unit mass of fuel and $c_{v}$ is the constant volume specific heat. Here $\mathrm{Da}$ is the Damköhler number, $\mathrm{Ze}$ is the Zel'dovich number, and $\mathrm{He}$ is the heat release parameter. Note that the molecular diffusivities $D_{i}$ and the thermal diffusivity $D_{T}$ can all be different. For the purposes of this study, we will assume that variations in the density and transport properties are sufficiently small that $G$ on the right-hand side of Eqs. 1 and 2 can be neglected. While restricting our present treatment to this somewhat simpler case, we believe that the ideas presented here are also applicable to the more general problem.

In a translating and rotating local coordinate frame moving with the reaction zone and remaining aligned with the diffusion layer, the velocity field in the vicinity of the origin is given by the pure strain flow $\left\{u_{n}, u_{s}, u_{t}\right\}=\left\{-\sigma n, \sigma_{s} s, \sigma_{t} t\right\}$, where $\sigma$ is the local time-varying strain rate along the layer normal direction. Equations 1 and 2 can then be shown to take the form

$$
\begin{gathered}
\frac{\partial c_{i}}{\partial t}-\left(v_{n}+\sigma n\right) \frac{\partial c_{i}}{\partial n}-\frac{1}{\operatorname{ReSc}} \frac{\partial^{2} c_{i}}{\partial n^{2}} \\
= \pm \nu_{i} \mathrm{Da}\left(c_{A} c_{B}\right) e^{-Z e / T}, \\
\frac{\partial T}{\partial t}-\left(v_{n}+\sigma n\right) \frac{\partial T}{\partial n}-\frac{1}{\operatorname{Re} \operatorname{Pr}} \frac{\partial^{2} T}{\partial n^{2}} \\
=\nu_{P} \operatorname{He} \mathrm{Da}\left(c_{A} c_{B}\right) e^{-Z e / T} .
\end{gathered}
$$

Here, gradients along the diffusion layer and non-inertial terms due to the moving coordinate frame have been presumed negligible. Also, since the reaction zone is not a material surface and can propagate relative to the fluid, we have without any loss of generality included the uniform normal velocity $v_{n}$ to allow the coordinate frame to follow the reaction zone in some appropriately defined sense. As of now $v_{n}$ is undetermined; we discuss its proper selection below.

\section{EVOLUTION EQUATIONS FOR THE PROFILE MOMENTS}

Assuming that the diffusion layer is sufficiently thin so that each variable and its normal derivatives in Eqs. 3 and 4 reach their far field value before encountering another layer, we can integrate these equations in the normal direction to obtain the moments of each profile. The physical nature of the reactant profiles far away from the diffusion layer renders their moments divergent, so we instead follow the evolution of the reactant gradient profiles. We define the moments of the gradient of the reactant profile $c_{i}$ as

$R_{j}^{i} \equiv \int_{-\infty}^{\infty} n^{j} \frac{\partial c_{i}}{\partial n} d n, \quad j=0,1,2, \ldots$

We assume here that all gradients go to zero sufficiently fast far away from the interface so that the moments are convergent. Obviously the zeroth moment, the integral of the gradient, is simply

$R_{0}^{i}=\mp c_{i}^{\infty}$,

where $c_{i}^{\infty}$ denotes the value of $c_{i}$ as $n \rightarrow \mp \infty$. Here, the sign on top (-) goes with $i=\{A\}$ and that on bottom (+) with $i=\{B\}$. We adopt this convention from here on, as there will be several cases where the expressions for the reactants are identical except for the sign. To obtain the evolution equation for the first moment $R_{1}{ }^{i}$, we differentiate Eq. 3 with respect to $n$, multiply by $n$, and integrate. The first term becomes

$$
\int n \frac{\partial}{\partial n} \frac{\partial c_{i}}{\partial t} d n=\frac{d}{d t} \int n \frac{\partial c_{i}}{\partial n} d n=\frac{d}{d t} R_{1}{ }^{i},
$$


the second term is

$$
\begin{aligned}
& \int n \frac{\partial^{2} c_{i}}{\partial n^{2}} d n \\
& \quad=\int\left(\frac{\partial}{\partial n} n \frac{\partial c_{i}}{\partial n}-\frac{\partial c_{i}}{\partial n}\right) d n= \pm c_{i}^{\infty},
\end{aligned}
$$

and the third term is

$$
\begin{aligned}
& \int n \frac{\partial}{\partial n} n \frac{\partial c_{i}}{\partial n} d n \\
& \quad=\int\left(\frac{\partial}{\partial n} n^{2} \frac{\partial c_{i}}{\partial n}-n \frac{\partial c_{i}}{\partial n}\right) d n=-R^{i} .
\end{aligned}
$$

The diffusion part is simply

$$
\begin{aligned}
& \int n \frac{\partial}{\partial n} \frac{\partial^{2} c_{i}}{\partial n^{2}} d n \\
& \quad=\int\left(\frac{\partial}{\partial n} n \frac{\partial^{2} c_{i}}{\partial n^{2}}-\frac{\partial^{2} c_{i}}{\partial n^{2}}\right) d n=0,
\end{aligned}
$$

and the reaction term becomes

$$
\begin{aligned}
\int & n \frac{\partial}{\partial n} e^{-Z e / T} c_{A} c_{B} d n \\
= & \int\left(\frac{\partial}{\partial n} n e^{-Z e / T} c_{A} c_{B}-e^{-Z e / T_{c}} c_{A} c_{B}\right) d n \\
= & -\int e^{-Z e / T} c_{A} c_{B} d n .
\end{aligned}
$$

In all cases we have integrated by parts and made use of the fact that the gradients vanish at infinity to obtain the final results. The evolution equations for higher moments are derived in a similar way. Collecting the results we have

$$
\begin{aligned}
\frac{d R_{0}{ }^{i}}{d t}= & 0, \\
\frac{d R_{1}^{i}}{d t}= & \pm v_{n} c_{i}^{\infty}-\sigma R_{1}^{i} \\
& +v_{i} \mathrm{Da} \int e^{-Z_{e} / T} c_{A} c_{B} d n, \\
\frac{d R_{2}{ }^{i}}{d t}= & -2 v_{n} R_{1}^{i}-2 \sigma R_{2}^{i} \mp 2 \frac{c_{i}^{\infty}}{\operatorname{ReSc}_{i}} \\
& +2 \nu_{i} \mathrm{Da} \int e^{-Z_{e} / T_{1}} c_{A} c_{B} n d n .
\end{aligned}
$$

The moments of the product profile, on the other hand, remain convergent and are defined as

$C_{j} \equiv \int_{-\infty}^{\infty} n^{j} c_{P} d n, \quad j=0,1,2, \ldots$

and the resulting evolution equations are given by $\frac{d C_{0}}{d t}=-\sigma C_{0}+\nu_{P} \mathrm{Da} \int e^{-Z e / T_{1}} c_{A} c_{B} d n$,

$$
\begin{aligned}
\frac{d C_{1}}{d t}= & -v_{n} C_{0}-2 \sigma C_{1} \\
& +\nu_{P} \mathrm{Da} \int e^{-Z e / T} c_{A} c_{B} n d n \\
\frac{d C_{2}}{d t}= & -2 v_{n} C_{1}-3 \sigma C_{2}+\frac{2 \bar{C}_{0}}{\operatorname{ReSc}} \\
& +v_{P} \mathrm{Da} \int e^{-Z e / T} c_{A} c_{B} n^{2} d n
\end{aligned}
$$

Similarly, for the temperature rise profile, the moments are denoted

$Q_{j} \equiv \int_{-\infty}^{\infty} n^{j}\left(T-T_{\infty}\right) d n, \quad j=0,1,2, \ldots$

and we have the evolution equations

$\frac{d Q_{0}}{d t}=-\sigma Q_{0}+\nu_{P} \mathrm{He} \mathrm{Da} \int e^{-Z e / T} c_{A} c_{B} d n$,

$$
\begin{aligned}
\frac{d Q_{1}}{d t}= & -v_{n} Q_{0}-2 \sigma Q_{1} \\
& +\nu_{P} \mathrm{He} \mathrm{Da} \int e^{-Z e / T} c_{A} c_{B} n d n \\
\frac{d Q_{2}}{d t}= & -2 v_{n} Q_{1}-3 \sigma Q_{2}+\frac{2 Q_{0}}{\operatorname{Re} \operatorname{Pr}} \\
& +\nu_{P} \mathrm{He} \mathrm{Da} \int e^{-Z e / r_{c_{A}} c_{B} n^{2} d n}
\end{aligned}
$$

Notice that, although Eqs. 5-7 still involve the temperature and reactant profiles explicitly, these profiles affect the layer evolution only through 
integrals resulting from the reaction rate terms. We refer to these as "reaction integrals." As a consequence, in order to accurately track the evolution of the profile moments and thereby determine global layer properties such as the burning rate, it is only necessary that these reaction integrals be evolved accurately. The point is that the integral nature of these terms suggests this may be possible even with relatively crude representations for the actual temperature and reactant profiles. It is this observation that we use the close the moment equations in the following section.

\section{IMPLEMENTATION}

Because the coordinate frame is to follow the reaction zone in some sense, we must now choose exactly what point to follow. The selection of this point determines $v_{n}$, and this can be done in several ways. Of course, one possibility is to take $v_{n}$ equal to zero, in which case the coordinate frame does not follow the reaction zone, but instead remains fixed to the material interface that initially separates the two reactants. In general, however, the reaction zone can move away from this interface, and if $v_{n}$ has been taken as zero an increase in the moments would account for the movement. As the distance increases, though, it is likely that the accuracy would deteriorate rapidly. A better choice is to select $v_{n}$ in such a way that the reaction zone remains fixed relative to the coordinate frame. One possibility is to insist that the coordinate frame follows the temperature profile in such a way that the first moment $Q_{1}$ vanishes. Then the normal velocity is given by

$v_{n}=\frac{1}{Q_{0}} \nu_{P} \mathrm{He} \mathrm{Da} \int e^{-Z e / T} c_{A} c_{B} n d n$,

and the equation for $Q_{1}$ drops out. Other selections are obviously possible.

The only approximations in the development up to this point are those leading to Eqs. 3 and 4, from which the evolution equations for the profile moments in Eqs. 5-7 were derived. However, to close these equations we must now make some assumptions about the temperature and reactant profiles that appear in the reaction integrals, and this is where it is necessary to invoke what is known about the structure of such layers. Experimental results of Dahm and Buch [24-26] show that the detailed internal structure of strained diffusion layers for conserved scalar mixing in turbulent flows is essentially self similar. The formal connection between the conserved scalar profile and the reactant, product and temperature profiles for a fast reaction with negligible heat release suggests that this self similarity will be present in reactive flows also. We therefore assume that each profile is self similar and can be adequately characterized by a family of shapes parameterized with just a few degrees of freedom. Once the family of profile shapes has been selected the moment equations are closed at each order, so no further assumptions are necessary.

Although the actual profiles will in general not be symmetric, and there is no great difficulty in incorporating such asymmetry, the use of symmetric profiles makes the algebra a little simpler and is consistent with the underlying theme of not attempting to accurately represent the detailed internal structure of the layer. Moreover, as is shown in the sections that follow, quite accurate results can be obtained even at this level of simplicity in modeling the actual profiles shapes. In practice, we have implemented two such families of profile shapes: (1) a simple "square" profile, for which the reactants vary linearly and the products and temperature are constant in a finite region, and (2) a smooth "algebraic" profile, which more nearly represents the expected shapes of the actual profiles.

\section{Square Profiles}

The square profiles, sketched in Fig. 1a, are defined as

$$
\frac{c_{i}(n)}{c_{i}^{\infty}}= \begin{cases}1, & \text { if } n \geqslant \Delta_{i}-\delta_{i} / 2 \\ 1 / 2, \pm\left(\Delta_{i}-n\right) / \delta_{i} & \text { if } \Delta_{i}-\delta_{i} / 2<n<\Delta_{i}+\delta_{i} / 2 \\ 0, & \text { if } n \leq \Delta+\delta_{i} / 2\end{cases}
$$



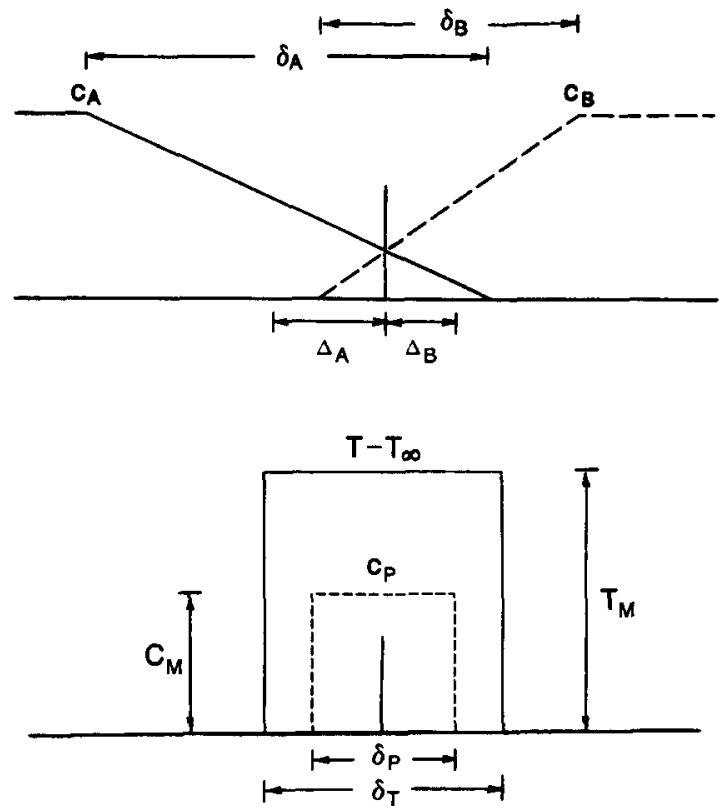

(a)
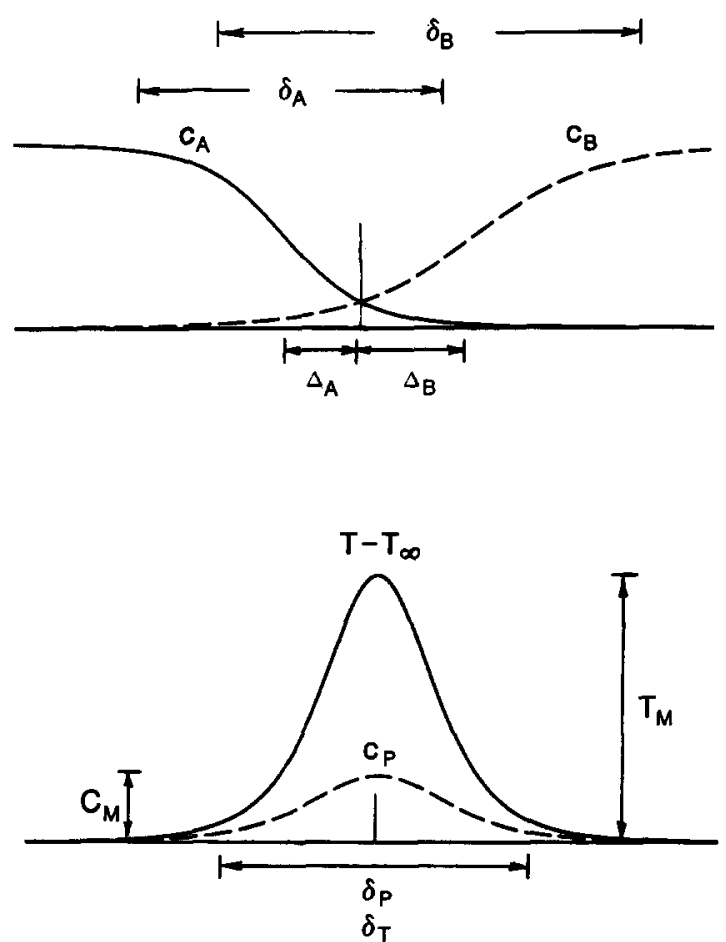

(b)

Fig. 1. Families of self-similar profile shapes used in the integral method. (a) Simple "square" profile shapes. (b) Smooth "algebraic" profile shapes.

$$
\begin{aligned}
& c_{P}(n)= \begin{cases}0, & \text { if } n \leq-\delta_{P} / 2 ; \\
C_{M}, & \text { if }-\delta_{P} / 2<n<\delta_{P} / 2 ; \\
0, & \text { if } n \geq \delta_{P} / 2 .\end{cases} \\
& T(n)-T_{\infty}= \begin{cases}0, & \text { if } n \leq-\delta_{T} / 2 ; \\
T_{M}, & \text { if }-\delta_{T} / 2<n<\delta_{T} / 2 ; \\
0, & \text { if } n \geq \delta_{T} / 2 .\end{cases}
\end{aligned}
$$

Here $\Delta_{i}$ are the displacements of the reactant gradient profiles from the origin, and $\delta_{i}$ are their widths. $T_{M}$ and $C_{M}$ are the uniform temperature rise and product concentration levels, respectively. Additionally, we have taken $v_{n}$ to be given by Eq. 8 so that the temperature profile is centered around the origin, and assumed that the product profile is also centered around this point. Each of the four profiles is therefore parameterized by two degrees of freedom; accordingly we must follow the evolution of two moments for each profile. These are related to the profile parameters as

$$
\begin{array}{ll}
R_{1}{ }^{i}=\mp c_{i}^{\infty} \Delta_{i} ; & R_{2}{ }^{i}=\mp c_{i}^{\infty}\left(\frac{\delta_{i}^{2}}{12}+\Delta_{i}^{2}\right) \\
C_{0}=C_{M} \delta_{P} ; & C_{2}=\frac{1}{12} C_{M} \delta_{P}{ }^{3} \\
Q_{0}=T_{M} \delta_{T} ; & Q_{2}=\frac{1}{12} T_{M} \delta_{T}^{3}
\end{array}
$$

Note that, for the square profiles, the reaction integrals in these equations can be calculated analytically.

\section{Algebraic Profiles}

To check the sensitivity of the method to the choice of imposed profile shapes, we have also implemented a family of smooth algebraic profiles, shown in Fig. 1b and defined as

$\frac{c_{i}(n)}{c_{i}^{\infty}}=\frac{1}{2}\left\{1 \mp I\left(n \pm \Delta_{i} ; \delta_{i}\right)\right\}$,

$c_{P}(n)=C_{M} J\left(n ; \delta_{P}\right)$,

$T(n)-T_{\infty}=T_{M} J\left(n ; \delta_{T}\right)$, 
where

$$
\begin{aligned}
& I\left(n ; \delta_{i}\right)=\frac{n^{3}+3 / 2 \delta_{i}^{2} n}{\left(n^{2}+\delta_{i}^{2}\right)^{3 / 2}} \\
& J\left(n ; \delta_{i}\right)=\frac{2}{3} \delta_{i} \frac{d I}{d n}=\frac{\delta^{5}{ }_{i}}{\left(n^{2}+\delta^{2}{ }_{i}\right)^{5 / 2}} .
\end{aligned}
$$

Each profile again has two degrees of freedom. In this case, the moments are

$$
\begin{array}{rlrl}
R_{1}^{i} & =\mp c_{i}^{\infty} \Delta_{i} ; & R_{2}^{i}=\mp c_{i}^{\infty}\left(\frac{\delta_{i}^{2}}{2}+\Delta_{i}^{2}\right), \\
C_{0}=\frac{4}{3} C_{M} \delta_{P}, & C_{2}=\frac{2}{3} C_{M} \delta_{P}^{3}, \\
Q_{0}=\frac{4}{3} T_{M} \delta_{T}, & Q_{2}=\frac{2}{3} T_{M} \delta_{T}{ }^{3} .
\end{array}
$$

For the smooth profiles, these integrals must be calculated numerically. For either family of profile shapes, Eqs. 5-7 determine the evolution of each of the degrees of freedom.

\section{RESULTS}

To test the accuracy of this integral method, we have applied it to a number of problems and compared it with results obtained using full finite difference calculations of Eqs. 3 and 4 . We have for simplicity taken all the diffusivities to be the same and given by $D$, and chosen as reference values $L^{*} \equiv \sqrt{D / \sigma}$ and $U^{*} / L^{*} \equiv \sigma$. The temperature is referenced to the far field value $T^{*} \equiv$ $T_{\infty}$, and the reactant and product concentrations to $c^{*}=c_{B}{ }^{\infty}$. This gives the nondimensional parameters as

$\mathrm{Da}=\frac{Z c_{B}^{\infty}}{\sigma}, \quad \mathrm{Ze}=\frac{E}{\mathrm{RT}_{\infty}}, \quad \mathrm{He}=\frac{q}{c_{v} T_{\infty}}$.

For convenience we have also divided the equation for $c_{A}$ by $c_{A}{ }^{\infty}$, so that the nondimensional concentration of both species at infinity is 1 . This simply requires that we replace $\nu_{B}$ with $\tilde{\nu}_{B} \equiv$ $\nu_{B}\left(c_{A}{ }^{\infty} / c_{B}{ }^{\infty}\right)$. In the calculations that follow, we have taken $\mathrm{Ze}=10$ and $\mathrm{He}=10$ to approximate typical hydrocarbon fuels. Lastly, we ignite the flame by taking as initial conditions $Q_{0}=1$ and $Q_{2}=1$.

\section{Isolated Diffusion Layers}

We consider first several cases of isolated strained diffusion and reaction layers under various strain rates. Figure 2 presents results for the time evolution of the integrated temperature rise across the layer, $Q_{0} \equiv \int\left(T-T_{\infty}\right) d n$, for an isolated layer with unity stoichiometry. Results from the present integral method in Eqs. 5-8, using both the square and smooth profiles, are shown and compared with the results from full finite difference calculations of Eqs. 3 and 4. For each of Figs. $2 \mathrm{a}-2 \mathrm{c}$ the strain rate history, $\sigma(t)$, experienced by the layer is somewhat different.

In Fig. 2a, the strain rate is constant and corresponds to $\mathrm{Da}=1000$. After ignition at $t=$ 0 , the integrated temperature initially rises until the dimensionless time $t \approx 1$, beyond which an equilibrium is reached where the continued generation and outward diffusion of heat and products within the layer is balanced by the attempts of the strain rate to reduce the layer thickness. During the initial phase, before this equilibrium is attained, the results obtained with the integral method using the smooth algebraic profiles agree well with the finite difference calculations. The results for the simple square profiles develop a little more slowly, presumably due to the lower peak temperature in that case (see also Fig. 3). However, once the strain-limited equilibrium is reached, the square profile attains virtually the correct steady state value while the smooth profile overestimates this value by slightly less than $7 \%$.

If the strain rate is now increased, one of two things can happen. In Fig. 2b, the Damköhler number is again held at $\mathrm{Da}=1000$ until $t=4$, but then the strain rate is suddenly increased fivefold so that $\mathrm{Da}=200$. In this case, the layer undergoes a similar transient before reaching a new strain-limited equilibrium at a somewhat lower value of the integrated temperature. Note that both the square and smooth profiles track this evolution quite well, though now the smooth profiles estimate the new steady state value a little 


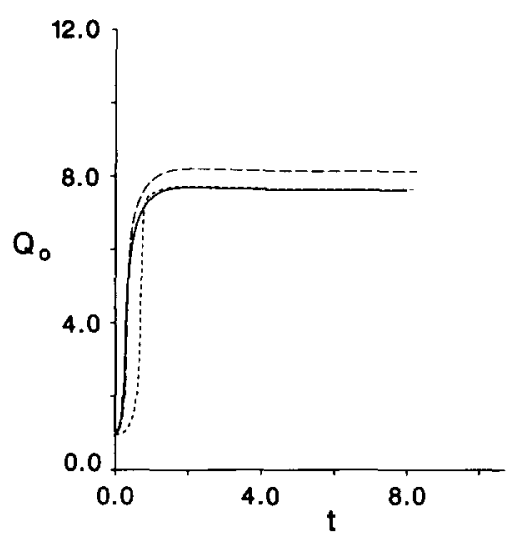

(a)

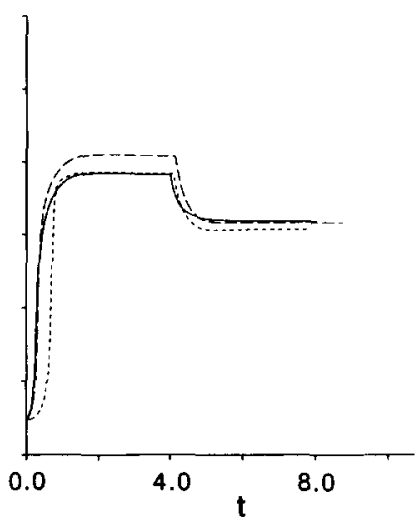

(b)

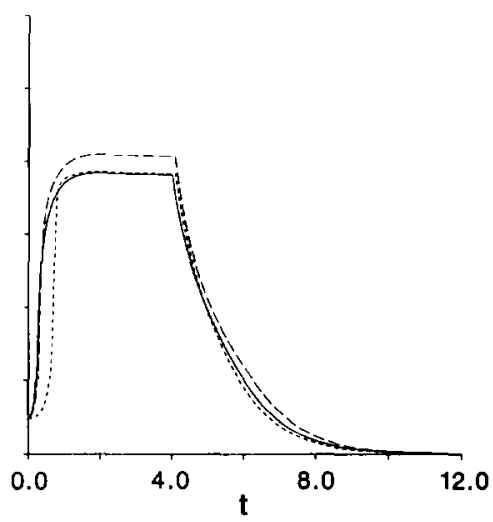

(c)

Fig. 2. Comparison of the integrated temperature rise across the diffusion layer as calculated by the integral method with results from full finite difference computations for the evolution of an isolated unsteady strained diffusion layer. In each case, the strain rate is held constant until the dimensionless time $t=4$, when it changes impulsively to the value indicated. finite differences; -----, integral method using square profiles, -----, integral method using smooth profiles. (a) $\mathrm{Da}=1000$, (b) $\mathrm{Da}=200$. (c) $\mathrm{Da}=50$.

more accurately. On the other hand, in Fig. 2c the strain rate at $t=4$ is increased by a factor of 20 , so that $\mathrm{Da}=50$. In this case the reaction in the layer abruptly extinguishes, since the heat generation can no longer keep up with the reduction in thickness due to straining. The integrated temperature then slowly decays to zero as the continued straining thins the residual product and temperature layer to zero thickness. Again, both the square and smooth profiles follow this extinction quite accurately.

It is remarkable how well the simple square profiles track the layer evolution in view of the fact that they only grossly represent the actual internal structure of the layer. This can be seen quite dramatically in Fig. 3, which shows the steady state profiles $c_{A}(n), c_{B}(n), c_{P}(n)$, and $T(n)$ at $\mathrm{Da}=200$ from Fig. $2 \mathrm{~b}$ for the finite difference calculations and the calculations with the integral method using both the smooth and square profile shapes. The actual profiles in Fig. $3 a$ are represented fairly accurately by the smooth profile shapes in Fig. 3b, though the widths are slightly narrower and the temperature is slightly higher. However, the square profile shapes in Fig. 3c resemble the actual profiles in Fig. 3a only in the crudest sense. Nevertheless, these square profiles still give values for the burning rate and extinction conditions in good agreement with the finite difference calculations. In fact there appears to be no consistent improvement achieved by using the smooth profiles over the simple square profiles. Evidently the smooth profiles, apart from being smooth, do not give inherently better approximations to the reaction integrals than do the simple square profiles.

An additional check on our method comes by considering the variation with Damköhler number of the steady-state value obtained for the integrated temperature rise across the layer. This result, shown in Fig. 4, yields the S-curve characteristic of large Zel'dovich number Arrhenius kinetics [16]. For high Damköhler numbers (low strain rates) a burning steady state results, while for low $\mathrm{Da}$ (large strain rates) the resulting steady state corresponds to local extinction. In between, there is a range of $\mathrm{Da}$ spanned by ignition and extinction limits where the final state depends on the strain rate history of the layer. Figure 4 shows the steady states obtained by the finite difference calculations for comparison with the integral method using both the square and smooth profiles. For both families of profiles shapes, the general form of the $\mathbf{S}$ curve is well reproduced, 


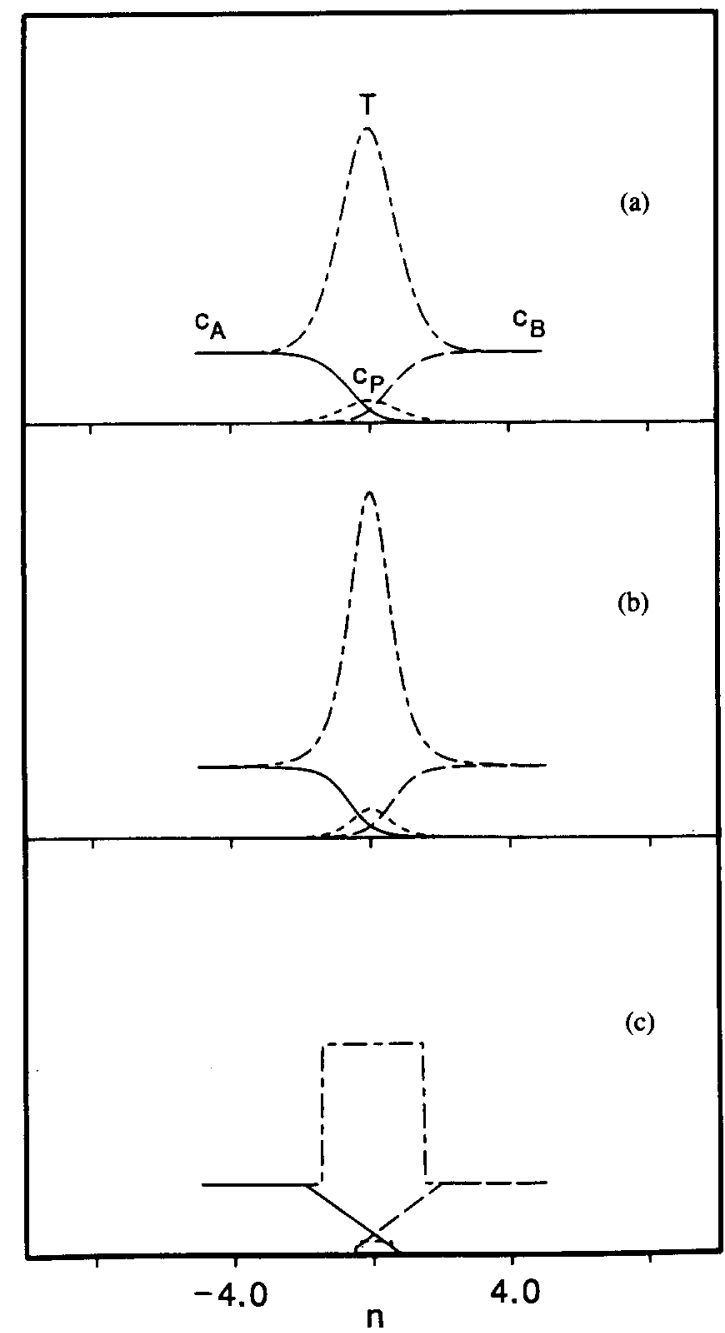

Fig. 3. Comparison of full finite difference results for the steady state profiles within the diffusion layer, shown here at $\mathrm{Da}=200$, with their simple representations used in the integral method. Although the family of square profile shapes used in the integral method only grossly represents the actual internal structure of the layer, they track the evolution of global layer properties as accurately as do the smooth profile shapes, typically within a few percent of the full finite difference results (a) Full finite difference calculations. (b) Integral method using smooth profiles. (c) Integral method using square profiles.

though the precise ignition and extinction limits vary slightly with the profile shape used. Note that the square profile actually gives better agreement with the finite difference calculations, the main difference being that its ignition limit lies at

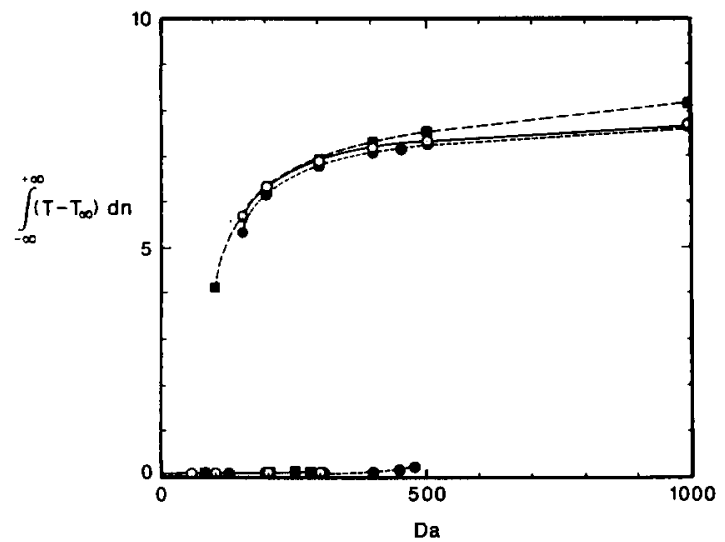

Fig. 4. Comparison of the steady-state integrated temperature rise across the diffusion layer versus Damköhler number, giving the $\mathbf{S}$ curve characteristic of large Zel'dovich number Arrhenius kinetics. $\bigcirc$, finite difference calculations; $\bullet$, integral method using smooth profiles; $\square$, integral method with square profiles.

a higher Da. As was noted in Figs. 2 and 3, this is presumably due to the lower maximum temperature for the square profile. The smooth profile, which generally has a higher maximum temperature than is seen in the finite difference calculations, gives both the ignition and extinction limits at slightly lower values of the Damköhler number.

We now turn to a case with stoichiometry significantly different from unity to demonstrate how the method works when the reaction zone moves away from the initial interface. Figure 5 shows the steady-state profiles for a reaction with $\tilde{\nu}_{B}=10$, approximating pure methane-air stoichiometry, and $\mathrm{Da}=2000$, with all other parameters the same as above. The reaction zone is now shifted toward the oxidizer (B) side, with an accompanying asymmetry in the reactant gradients. Note that the origin shown is the original interface. Because a large amount of inert components are now carried with the oxidizer, the overall temperature is considerably lower than in the previous case. The finite difference calculations show an asymmetric temperature profile, but in our integral method we have elected for simplicity to use a symmetric profile. This causes the maximum temperature to lie somewhat off the overlap region for the reactants and therefore 


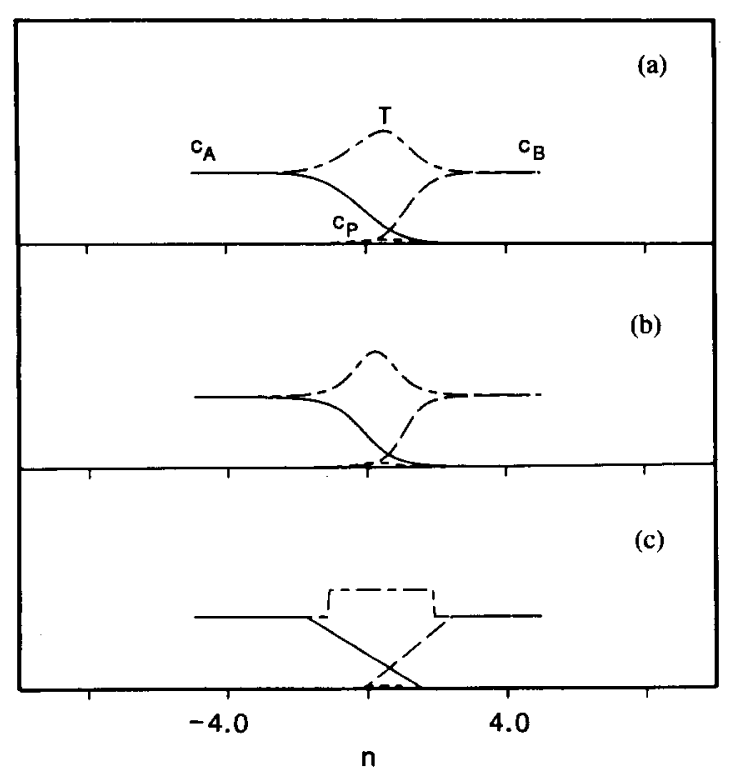

Fig. 5. Steady state profile shapes at $\mathrm{Da}=2000$ for non-unity stoichiometry; $\tilde{\nu}_{B}=10$. (a) Full finite difference calculations. (b) Integral method using smooth profiles. (c) Integral method using square profiles.

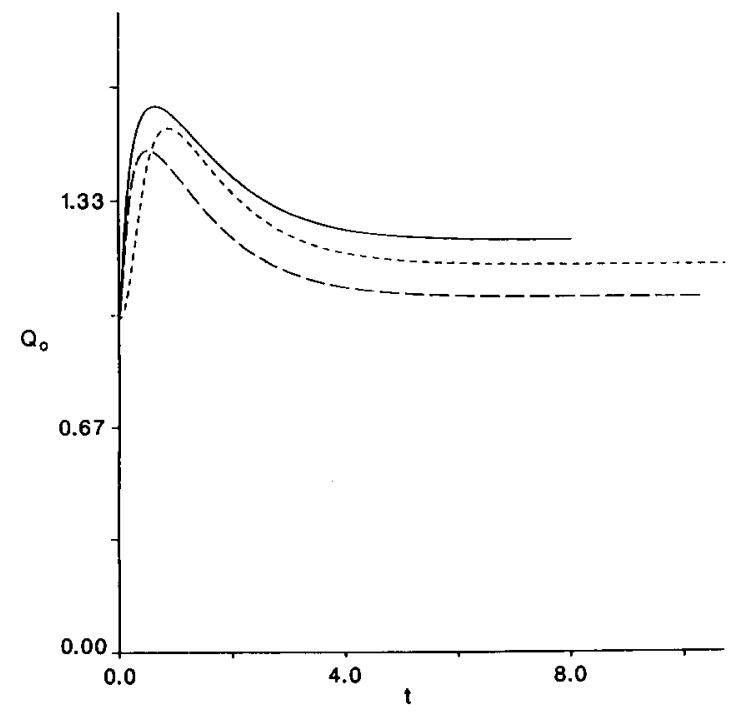

Fig. 6. Evolution of the integrated temperature rise across the diffusion layer for $\mathrm{Da}=2000, \tilde{\nu}_{B}=10$. - , finite differences; -----, integral method using square profiles -----, integral method using smooth profiles. Note that the nature of the square profiles in Fig. 5 gives better agreement with the full finite difference results for the global layer properties than do the smooth profiles.

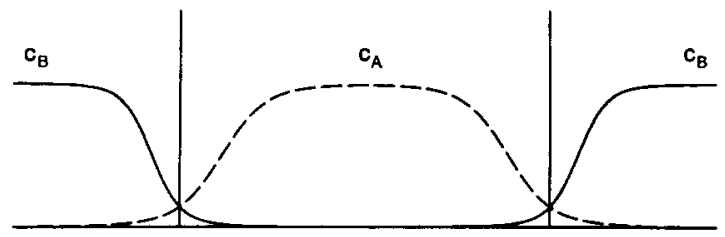

Fig. 7. Interaction of two parallel strained diffusion layers.

leads to a slightly lower reaction rate. Nevertheless, even with such a simple representation of the actual profile shapes in the reaction integrals, the integrated temperature across the layer for the square profiles is still within $6 \%$ of the actual value. This is reflected in Fig. 6, showing the time evolution of the integrated temperature. Again the simple square profile is somewhat more accurate than the smooth profile. This can be understood in Fig. 5 by comparing the overlap of the temperature profile with the product of the reactant profiles, which drives the layer evolution through the reaction integrals. The nature of the square temperature profile renders it less sensitive to the asymmetry than is the case for the smooth profile.

\section{Interacting Diffusion Layers}

We now consider the evolution of two parallel strained diffusion layers bounding a fuel strip (Fig. 7) to illustrate how the method can be extended to treat interacting diffusion layers. Because the derivation of the moment equations assumed an isolated diffusion layer, the extension to interacting layers requires some additional approximations. We begin by noting that, except for the reaction integrals, the moment equations are linear. This suggests that we might construct each of the reactant profiles as a linear superposition of two profiles, namely $c_{A}=1-\left(c_{A}{ }^{L}-\right.$ $\left.c_{A}{ }^{R}\right)$ and $c_{B}=\left(c_{B}{ }^{L}+c_{B}{ }^{R}\right)$, where $L$ and $R$ denote the left- and right-hand sides of the fuel strip, and evolve them according to Eqs. 5-7. In the absence of chemical reactions the equations governing the evolution of each of the four component profiles would be completely decoupled. However, when a reaction is present the component profiles for the left- and right-hand layers are coupled through the reaction integrals. Here 


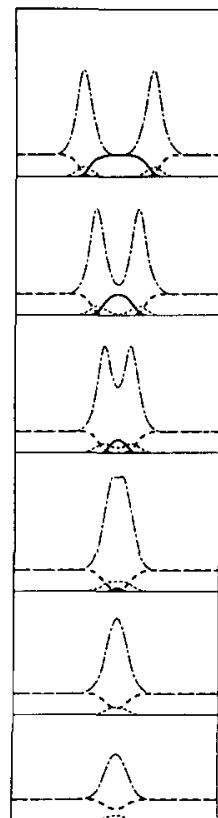

(a) (b)

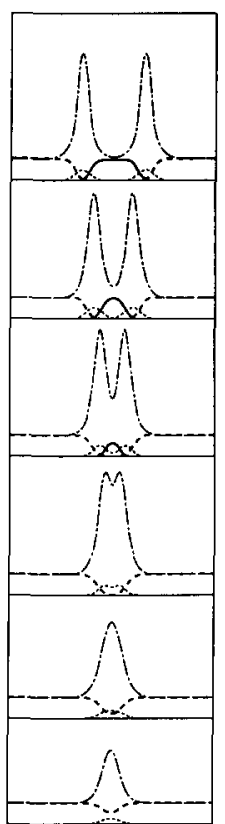

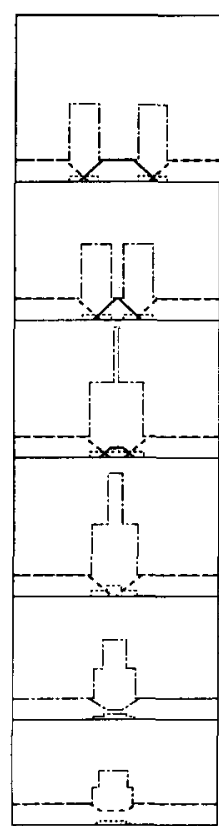

(c)
Fig. 8. Time sequence showing the evolution of mixing and chemical reaction in two parallel interacting strained diffusion layers at $\mathrm{Da}=1000$. See also Fig. 9. The first frame is at $t=2$, and subsequent frames are at time intervals of 0.5 . (a) Full finite difference calculations. (b) Integral method using smooth profiles. (c) Integral method using square profiles.

we simply divide the integration range for the reaction integrals into two parts, one spanning from $-\infty$ to the center of the strip and affecting only the left-hand side profiles, and the other from the center to $+\infty$ affecting the right-hand profiles only. Note that this is precisely correct for the symmetric burning that results if both layers are at the same stoichiometry. If the two layers have unequal stoichiometries, this can be approximately incorporated by splitting the integration range at a point off the centerline.

A sequence of profiles for a finite difference calculation and the integral method with both the smooth and square profiles is shown in Fig. 8. The corresponding evolution of the integrated temperature across the layer is shown in Fig. 9. Here the strain rate is held constant at $\mathrm{Da}=1000$, with all other parameters the same as in the previous cases. In the first frame, corresponding to the dimensionless time $t=2.0$, each layer is

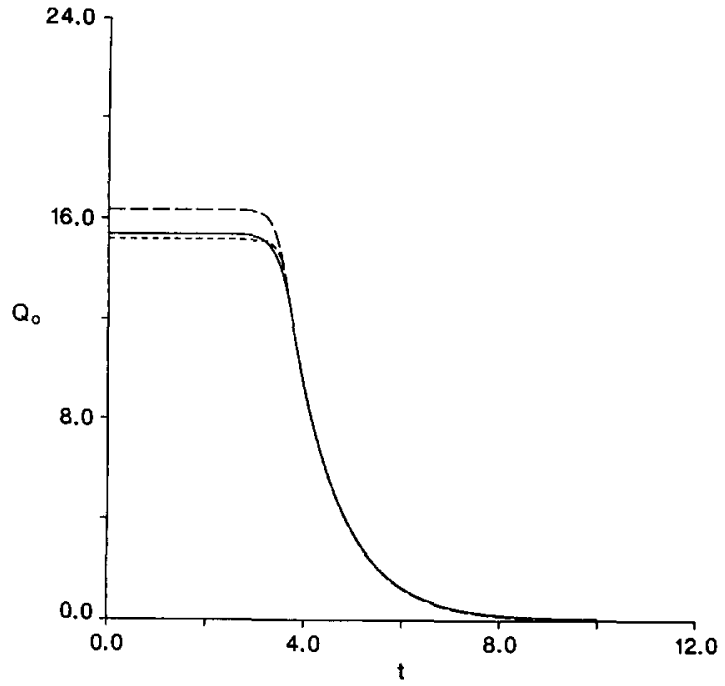

Fig. 9. Evolution of the integrated temperature rise across the interacting diffusion layers for the calculations shown in Fig. 8. - - , finite difference; -----, integral method using square profiles; -..--, integral method using smooth profiles.

at steady state. At this relatively early stage, the reaction layers are essentially independent of each other and move only due to the strain field. In the second frame the layers have just begun to interact. By the third frame, however, considerable interaction has taken place, with the remaining fuel being rapidly consumed. At the fourth frame, the fuel strip has been nearly consumed. Beyond this point the oxidizer profiles continue to diffuse into each other as the heat is reduced by straining (fifth and sixth frames). Note in Fig. 9 that the square profiles, despite the obvious differences in the internal layer structure, nevertheless capture the evolution as well as the more complicated smooth profiles. Indeed both sets of profiles follow very well the consumption of the fuel strip.

\section{CONCLUSIONS}

We have introduced a simple integral method for determining the evolution of molecular mixing and finite rate chemical reactions in diffusion layers under the effects of an arbitrarily timevarying strain rate. The governing partial differential equations for the reactants, product and temperature are used to derive ordinary differen- 
tial equations for the evolution of the moments of these profiles. These equations are remarkable in the sense that the actual profile shapes are seen to affect the layer evolution only through integrals resulting from the reaction rate terms. As a result, it is not necessary that these profiles be precisely known in order to accurately track the evolution of global properties of the layer. Indeed, very simple representations for these profiles with just a few degrees of freedom, such as the family of square profiles for which the reaction integrals can even be evaluated analytically, are quite sufficient to determine quantities such as the local burning rate and extinction conditions to within a few percent. This is certainly comparable to the accuracy typical of integral methods used in determining the evolution of boundary layers under the effects of arbitrary pressure gradients.

The approach described here can be immediately extended in several ways. More complex kinetic representations, such as multiple step reactions, simply require tracking a larger number of species coupled through their reaction integrals. For higher-order reactions, as well as nonArrhenius kinetics, only the form of the reaction integrals will change, with the method remaining the same. We also believe that the method could be extended to account for the effects of fluid expansion due to heat release, in which case $G$ on the right-hand sides of Eqs. 1 an 2 is no longer negligible. This would require a coupling back from the reaction to the flow field through a source distribution in the reaction zone. Similarly, effects of differential diffusion are easily accounted for by allowing a different Schmidt number for each species in Eqs. 5-7, and likewise nonuniform transport properties can also be included in $G$.

Although this method may be useful for studies of certain aspects of the simple one-dimensional, time-dependent diffusion and reactions problems demonstrated here, its real utility is expected to be as a subscale model in calculations of turbulent diffusion flames in situations where the relevant Damköhler number is sufficiently large for the flamelet assumption to be valid. The need for full reacting flow simulations to computationally re- solve the scales on which molecular diffusion and reactions occur, which are generally much smaller than the hydrodynamic scales, makes the resolution requirements for reactive turbulent flows more severe than for nonreacting flows. Representing these diffusion and reaction layers with an integral method such as the one presented here reduces the resolution requirements to what is required for nonreacting flows. Treatment of the combustion dynamics is therefore reduced to following a single interface, much as in the thinflame Burke-Schmann model, but now allows the effects of time-varying strain rates and finite rate kinetics, including extinction, to be retained.

We would like to acknowledge useful discussions with Professor Robert Krasny in the Applied Mathematics group at Michigan, as well as the help of Mr. Chester Chang in checking some of the calculations and in preparation of the figures. The work reported here was supported, in part, by the Gas Research Institute (GRI) under Contract Nos. 5087-260-1443 and 5088-260-1692, and by the Air Force Office of Scientific Research (AFOSR) under Grant No. AFOSR-89-0541.

\section{REFERENCES}

1. Ashurst, Wm. T., Kerstein, A. R., Kerr, R. M., and Gibson, C. H., Phys. Fluids 30:2343-2353 (1987).

2. Gibson, C. H., Ashurst, Wm. T., and Kerstein, A. R., J. Fluid Mech. 194:261-293 (1989).

3. Marble, F. E., and Broadwell, J. E., Project SQUID Tech. Rep. TRW-9-PU, 1977.

4. Gibson, C. H., and Libby, P. A., Combust. Sci. Technol. 8:29-35 (1972).

5. Liew, S. K., Bray, K. N. C., and Moss, J. B., Combust. Sci. Technol. 27:69-73 (1981).

6. Peters, N., and Williams, F. A., AIAA J. 21:423-429 (1983).

7. Peters, N., Prog. Energ. Combust. Sci. 10:319-339 (1984).

8. Peters, N., Twenty-First Symposium (International) on Combustion, The Combustion Institute, Pittsburgh, 1986, pp. 1231-1250.

9. Pope, S. B., and Cheng, W. K., Twenty-Second Symposium (International) on Combustion, The Combustion Institute, Pittsburgh, 1988.

10. Broadwell, J. E., and Breidenthal, R. E., J. Fluid Mech. 125:397-410 (1986). 
11. Dimotakis, P. E., GALCIT Report FM87-1, Caltech, Pasadena, CA, 1987.

12. Broadwell, J. E., and Mungal, M. G., Twenty-Second Symposium (International) on Combustion, The Combustion Institute, 1988, pp. 579-587.

13. Broadwell, J. E., Proceedings of the US-France Workshop on Turbulent Reactive Flows, 1987.

14. Burke, S. P., and Schumann, T. E. W., Ind. Eng. Chem. 20:988-1004 (1928).

15. Fendell, F. E., J. Fluid Mech. 21:281-303 (1965).

16. Williams, F. A., AIAA J. 24:867-875 (1986).

17. Liñán, A., Acta Astronaut. 1:1007-1039 (1974).

18. Dixon-Lewis, G., David, T., Gaskell, P. H., Fukutani, S., Jinno, H., Miller, J. A., Kee, R. J., Smooke, M. D., Peters, N., Effelsberg, E., Warnatz, J., and Behrendt, F., Twentieth Symposium (International) on Combustion, The Combustion Institute, Pittsburgh, 1984, pp. 1893-1904.

19. Carrier, G. F., Fendell, F. E., and Marble, F. E., SIAM J. Appl. Math. 28(2):463-500.
20. Marble, F. E., in Recent Advances in Aerospace Sciences (C. Casci, Ed.) Plenum, New York, p. 395.

21. Haworth, D. C., Drake, M. C., Pope, S. C., and Blint, R. J., Twenty-Second Symposium (International) on Combustion, The Combustion Institute, Pittsburgh, 1988, pp. 589-597.

22. White, F. M., Viscous Fluid Flow, McGraw-Hill, New York, 1974.

23. Williams, F. A., Combustion Theory, Benjamin/Cummings, Menlo Park, CA, 1985.

24. Dahm, W. J. A., and Buch, K. A., Turbulent Shear Flows 7, Springer Verlag, in press.

25. Dahm, W. J. A., Southerland, K. B., and Buch, K. A., Applications of Laser Methods to Fluid Mechanics, Springer Verlag, Berlin, in press.

26. Dahm, W. J. A., and Buch, K. A., J. Fluid Mech. (submitted).

Received II November 1989; revised II June 1990 